Europhysics Letters

PREPRINT

\title{
Giant nonlinearity and entanglement of single photons in photonic bandgap structures
}

\author{
I. Friedler ${ }^{1}$, G. Kurizki ${ }^{1}$ and D. Petrosyan ${ }^{2}$ \\ 1 Department of Chemical Physics, Weizmann Institute of Science, Rehovot 76100, \\ Israel \\ 2 Institute of Electronic Structure \& Laser, FORTH, Heraklion 71110, Crete, Greece
}

PACS. 03.67.-a - Quantum information.

PACS. 42.70.Qs - Photonic bandgap materials.

PACS. 42.50.Gy - Effects of atomic coherence on propagation, absorption, and amplification of light; electromagnetically induced transparency and absorption.

\begin{abstract}
Giantly enhanced cross-phase modulation with suppressed spectral broadening is predicted between optically-induced dark-state polaritons whose propagation is strongly affected by photonic bandgaps of spatially periodic media with multilevel dopants. This mechanism is shown to be capable of fully entangling two single-photon pulses with high fidelity.
\end{abstract}

Introduction. - The main impediment towards the use of single photons in schemes for deterministic quantum logic and teleportation [1] as very robust and versatile carriers of quantum information [2] is the weakness of optical nonlinearities in conventional media. A major trend aimed at the enhancement of optical nonlinearities exploits one-dimensional (1D-) periodic distributed Bragg reflectors and 2D- or 3D-periodic photonic crystals (PCs), where light can be slowed down or trapped via multiple reflections in the vicinity of photonic bandgaps (PBGs) [3]. Giantly enhanced nonlinearity has been predicted when dopants with transition frequencies within the PBG are implanted in the structure, so that light near such frequencies resonantly interacts with the dopants and is concurrently affected by the PBG dispersion [4]. The resulting soliton-like transmission of very weak pulses within the PBG while filtering out spurious noise is highly advantageous for classical communication [5]. However, this mechanism is incompatible with the goals of quantum logic and communications, particularly with photon-photon entanglement, because of the quantum noise associated with resonant field-atom interactions.

Another pathway to enhance the nonlinearities is based on electromagnetically induced transparency (EIT) in atomic media, which comes about when classical driving fields induce coherence between atomic levels and transform the weak fields into atom-dressed dark-state polaritons [6,7]. The ultrahigh sensitivity of the EIT polaritonic dispersion to a small fieldinduced Stark shift of its atomic level can result in an appreciable nonlinear phase shift, impressed by one ultraweak field upon another [8]. Notwithstanding this promising sensitivity, EIT-polariton entanglement by a large conditional phase shift of one photon in the presence of another (also known as cross-phase modulation) faces serious challenges in spatially uniform

(c) EDP Sciences 
(a)

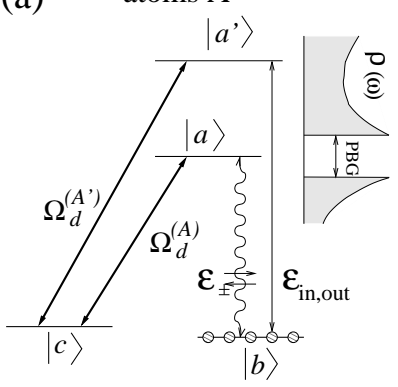

atoms B

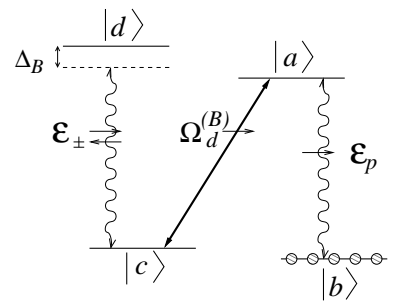

(c)
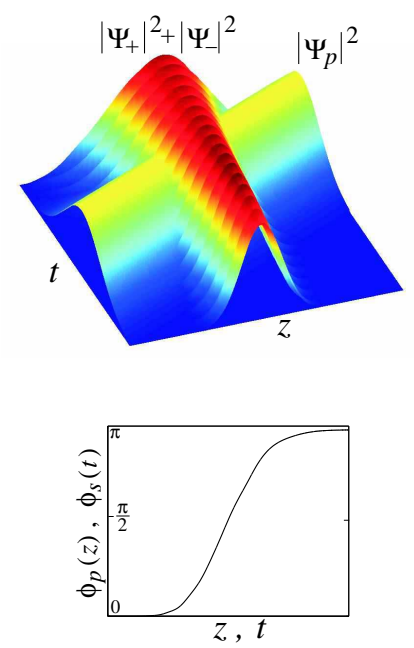

(b)

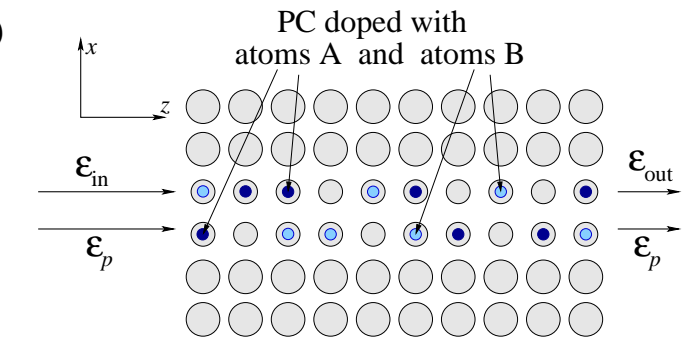

PC doped with

Fig. 1 - (a) Level scheme of atomic species A and B. Atoms A convert the input signal field $\mathcal{E}_{\text {in }}$ at frequency outside the PBG to the trapped field $\mathcal{E}_{ \pm}$at frequency inside the PBG. Atoms B provide EIT for the probe field $\mathcal{E}_{p}$ and its cross-coupling with the signal field $\mathcal{E}_{ \pm}$. (b) 2D-periodic photonic crystal (PC) having the density of modes $\rho(\omega)$, is doped with atomic species A and B. (c) Interaction and the resulting phase-shift of the probe and signal pulses.

media. One drawback of these schemes has been the mismatch between the group velocities of the probe pulse moving as a slow EIT polariton and the nearly-free propagating signal pulse, which severely limits their effective interaction length and the maximal conditional phaseshift [9]. To enable long interaction times and thus large conditional phase shifts in a medium of finite length (up to a few centimeters), the group velocities of both interacting pulses should be small $[10,11]$. This, however, imposes a limitation on the photonic component of the signal polariton whose magnitude determines the phase shift. Copropagating pulses pose yet another difficulty: since the phase-shift of each pulse is proportional to the local intensity of the other pulse, different parts of the interacting pulses acquire different phase shifts, which results in their spectral broadening.

Here we put forward a mechanism that may produce strong photon-photon interactions along with suppressed quantum noise and give rise to their entanglement with high fidelity, by combining the advantages of their dispersion in PBG structures and of the strongly enhanced nonlinear optical coupling achievable via EIT in an appropriately doped medium. The main idea is that a single-photon signal pulse is adiabatically converted into a standing-wave polaritonic excitation inside the periodic structure. This trapped polariton, having an appreciable photonic component, can impress a large, spatially-uniform phase shift upon a slowly propagating probe polariton. This task can further be facilitated by employing $2 \mathrm{D}$ - or 3D-periodic structures with defects where the two pulses interact via tightly confined modes $[3,12,13]$.

Photon-photon interaction in PBG structures. - The proposed scheme is based upon a periodic structure containing uniformly distributed dopants - atoms A and B (see fig. 1). Atoms A, having double- $\Lambda$ level configuration and interacting with classical driving fields on 
the transitions $|c\rangle_{A} \rightarrow|a\rangle_{A},\left|a^{\prime}\right\rangle_{A}$, with the Rabi frequencies $\Omega_{d}^{\left(A, A^{\prime}\right)}$, respectively, facilitate the trapping of the signal pulse inside the periodic structure, by converting its frequency from outside to inside of the PBG. On the other hand, atoms $\mathrm{B}$, having $\mathrm{N}$ level configuration and interacting with the $\Omega_{d}^{(B)}$ driving field on the transition $|c\rangle_{B} \rightarrow|a\rangle_{B}$, serve to simultaneously slowdown the probe pulse and cross-couple it with the signal.

The following procedure is foreseen to this end. Initially, all atoms A are in the ground state $|b\rangle_{A}$, the driving fields $\Omega_{d}^{(A)}=0$ and $\Omega_{d}^{\left(A^{\prime}\right)}>T_{\text {in }}^{-1}$, where $T_{\text {in }}$ is the temporal width of the input signal pulse $\mathcal{E}_{\text {in }}$. The carrier frequency of $\mathcal{E}_{\text {in }}$ is outside the $\mathrm{PBG}$, close to the $|b\rangle_{A} \rightarrow\left|a^{\prime}\right\rangle_{A}$ transition frequency, so that the usual EIT for the input signal due to the $\Lambda$ configuration $|b\rangle_{A} \leftrightarrow\left|a^{\prime}\right\rangle_{A} \leftrightarrow|c\rangle_{A}$ is realized. Upon entering the medium, the signal pulse is slowed down and spatially compressed, by a factor of $v_{s}^{\prime} / c \ll 1$, to the length $z_{\text {loc }} \simeq T_{\text {in }} v_{s}^{\prime}$, where $v_{s}^{\prime} \propto\left|\Omega_{d}^{\left(A^{\prime}\right)}\right|^{2}$ is its group velocity inside the medium. Once the signal pulse has fully accommodated in the medium of length $L$, which requires that $z_{\text {loc }}<L$, the driving field $\Omega_{d}^{\left(A^{\prime}\right)}$ is adiabatically switched off. As a result, the signal is stopped, its photonic component being converted into the stationary atomic (Raman) coherence $\sigma_{b c}^{(A)}[6,7]$. Next, the driving field $\Omega_{d}^{(A)}$ is adiabatically switched on to a value $\Omega_{d}^{(A)}>\Omega_{d}^{\left(A^{\prime}\right)}$, converting the atomic coherence into the signal field $\mathcal{E}_{ \pm}$, whose frequency is inside of the PBG and amplitude is larger than that of the input signal $\mathcal{E}_{\text {in }}$ by a factor of $\sqrt{v_{s} / v_{s}^{\prime}} \simeq \Omega_{d}^{(A)} / \Omega_{d}^{\left(A^{\prime}\right)}>1$ [7]. Due to the Bragg scattering of the forward and backward propagating components of the signal pulse with the wave vectors $\pm k$, it remains localized (trapped) within the medium [3]. Both components $\mathcal{E}_{ \pm}$of the signal field dispersively interact with atoms $\mathrm{B}$ via transition $|c\rangle_{B} \rightarrow|d\rangle_{B}$ with the detuning $\Delta_{B}$. This off-resonant interaction causes an ac Stark shift of level $|c\rangle_{B}$, thereby strongly affecting the EIT dispersion for the probe field $\mathcal{E}_{p}$, which interacts with atoms B on the transition $|b\rangle_{B} \rightarrow|a\rangle_{B}$ [8]. For a large enough product of the signal-field intensity $\left|\mathcal{E}_{ \pm}\right|^{2}$ and interaction time $L / v_{p}$ ( $v_{p}$ being the probe group velocity), both pulses accumulate a uniform conditional phase-shift which can reach $\pi$ (see fig. 1(c)). Finally, reversing the sequence that resulted in trapping of the signal pulse, its frequency is converted back to the original frequency and the $\mathcal{E}_{\text {out }}$ pulse leaves the medium.

Let us now consider the scheme more quantitatively. To describe the quantum properties of the medium, we use collective slowly varying atomic operators $\hat{\sigma}_{\mu \nu}^{(\iota)}(z, t)=\frac{1}{N_{\iota}^{z}} \sum_{j=1}^{N_{\iota}^{z}}\left|\mu_{j}\right\rangle_{\iota \iota}\left\langle\nu_{j}\right| e^{-i \omega_{\mu \nu}^{(\iota)} t}$, averaged over a small but macroscopic volume containing many dopants of species $\iota=A, B$ around position $z[6]: N_{A, B}^{z}=\left(N_{A, B} / L\right) \mathrm{d} z \gg 1$, where $N_{A, B}$ is the total number of the corresponding dopants. The quantum radiation is described by the traveling-wave (multimode) electric field operators $\hat{\mathcal{E}}_{ \pm}(z, t)=\sum_{q} a_{ \pm}^{q}(t) e^{ \pm i q z}$ and $\hat{\mathcal{E}}_{p}(z, t)=\sum_{q} a_{p}^{q}(t) e^{i q z}$, where $a_{j}^{q}$ are the annihilation operators for the field mode with the wave-vector $k_{j}+q, k_{j}$ being the carrier wave vector of the corresponding field. These single-mode operators possess the standard bosonic commutation relations $\left[a_{i}^{q}, a_{j}^{q^{\prime} \dagger}\right]=\delta_{i j} \delta_{q q^{\prime}}$, which yield $\left[\hat{\mathcal{E}}_{i}(z), \hat{\mathcal{E}}_{j}^{\dagger}\left(z^{\prime}\right)\right]=L \delta_{i j} \delta\left(z-z^{\prime}\right)$.

Using the standard technique [6], we perturbatively solve the Heisenberg equations for the atomic coherences $\hat{\sigma}_{\mu \nu}^{(\iota)}$ under EIT conditions [6-11] and substitute the solution into the propagation equations for the slowly varying field operators $\hat{\mathcal{E}}_{j}(z, t)$. These operators are related to the polariton operators $\hat{\Psi}_{ \pm}=\hat{\mathcal{E}}_{ \pm} / \cos \theta_{A}$ and $\hat{\Psi}_{p}=\hat{\mathcal{E}}_{p} / \cos \theta_{B}$, which represent the coupled excitation of the corresponding field and atomic Raman coherence $\hat{\sigma}_{b c}^{(\iota)}$. The mixing angles $\theta_{A, B}$ are defined via $\tan \theta_{A, B}=g_{A, B} \sqrt{N_{A, B}} / \Omega_{d}^{(A, B)}$, where $g_{A, B}=\wp_{a b}^{(A, B)} \sqrt{\omega_{a b}^{(A, B)} /\left(2 \hbar \epsilon_{0} S L\right)}$ are the atom-field coupling constants, $\wp_{\mu \nu}^{(\iota)}$ being the corresponding atomic dipole matrix element and $S$ the cross-sectional area of the quantum fields. Note that the amplitude of the 
photonic component of each polariton is proportional to the $\cos \theta_{\iota}$ of the corresponding mixing angle $\theta_{\iota}$. Under the Bragg resonance condition $k \simeq \pi / p_{s}$, where $p_{s}$ is the structure period, the equations of motion for polaritons are obtained as

$$
\begin{aligned}
& \left(\frac{\partial}{\partial t} \pm v_{s} \frac{\partial}{\partial z}\right) \hat{\Psi}_{ \pm}=-\kappa_{s} \hat{\Psi}_{ \pm}+i \eta \hat{I}_{p} \hat{\Psi}_{ \pm}+i \beta \hat{\Psi}_{\mp}+\hat{\mathcal{F}}_{s} \\
& \left(\frac{\partial}{\partial t}+v_{p} \frac{\partial}{\partial z}\right) \hat{\Psi}_{p}=-\kappa_{p} \hat{\Psi}_{p}+i \eta \hat{I}_{s} \hat{\Psi}_{p}+\hat{\mathcal{F}}_{p} .
\end{aligned}
$$

Here $v_{s}=c \cos ^{2} \theta_{A}$ and $v_{p}=c \cos ^{2} \theta_{B}$ are the group velocities, $\hat{I}_{s} \equiv \hat{\Psi}_{+}^{\dagger} \hat{\Psi}_{+}+\hat{\Psi}_{-}^{\dagger} \hat{\Psi}_{-}$and $\hat{I}_{p} \equiv \hat{\Psi}_{p}^{\dagger} \hat{\Psi}_{p}$ are the intensities (excitation numbers) of the signal and probe polaritons, respectively; $\kappa_{s, p}=\gamma_{b c}^{(A, B)} \sin ^{2} \theta_{A, B}$ are the absorption rates, $\gamma_{b c}^{(\iota)}$ being the Raman coherence decay rate of the corresponding atoms, $\hat{\mathcal{F}}_{s, p}$ are the $\delta$-correlated noise operators associated with the relaxation; $\eta=\left[1+i \gamma_{d}^{(B)} /\left(2 \Delta_{B}\right)\right] \cos ^{2} \theta_{A} \sin ^{2} \theta_{B} g_{B}^{\prime 2} / \Delta_{B}$ is the cross-coupling rate between the polaritons, $g_{B}^{\prime}=\wp_{d c}^{(B)} \sqrt{\omega_{d c}^{(B)} /\left(2 \hbar \epsilon_{0} S L\right)}$ being the atom-field coupling on the transition $|c\rangle_{B} \rightarrow|d\rangle_{B}$ and $\gamma_{d}^{(B)}$ the decay rate of $|d\rangle_{B}$ (for $\Delta_{B} \gg \gamma_{d}^{(B)}$, the cross-absorption vanishes [8-10] and then $\eta$, being purely real, represents the cross-phase modulation rate). Finally, $\beta=\frac{1}{2} \delta \omega_{\mathrm{PBG}} \cos ^{2} \theta_{A}$ is the Bragg reflection rate, $\delta \omega_{\mathrm{PBG}}$ being the PBG bandwidth.

Equations (11) constitute the starting point of our analysis. Their general solution, for arbitrary initial/boundary conditions of the traveling-wave quantized polaritons $\hat{\Psi}_{p, \pm}$ is not known. When absorption is negligible (see below), for a given time- and space-dependence of the signal-polariton intensity $\hat{I}_{s}(z, t)$, the solution for the probe is

$$
\hat{\Psi}_{p}(z, t)=\hat{\Psi}_{p}(0, \tau) \exp \left[i \frac{\eta}{v_{p}} \int_{0}^{z} \hat{I}_{s}\left(z^{\prime}, \tau+z^{\prime} / v_{p}\right) \mathrm{d} z^{\prime}\right],
$$

where $\tau=t-z / v_{p}$ is the retarded time. An analytic solution for the two counter-propagating components of the signal polariton can be obtained in the long probe limit, i.e., when the spatial dependence of the probe-polariton intensity is negligible on the scale of $z_{\text {loc }}: \hat{I}_{p}(z, t) \simeq$ $\hat{I}_{p}(t)$. This requires that $v_{p} T_{p}>z_{\text {loc }}$, where $T_{p}$ is the duration of the probe pulse (its spectral width being $\delta \omega_{p} \sim T_{p}^{-1}<v_{p} / z_{\text {loc }}$ ). Then eq. (1a) is soluble by the Fourier transform $\hat{\Psi}_{ \pm}(z, t)=\frac{1}{2 \pi} \int \mathrm{d} q e^{ \pm i q z} \hat{\psi}_{ \pm}(q, t)$, with the result

$$
\begin{aligned}
& \hat{\psi}_{+}(q, t)=\hat{\psi}_{+}(q, 0) e^{i \hat{\phi}_{s}(t)}\left[\cos (\chi t)-i \frac{q v_{s}}{\chi} \sin (\chi t)\right], \\
& \hat{\psi}_{-}(q, t)=i \hat{\psi}_{+}(-q, 0) e^{i \hat{\phi}_{s}(t)} \frac{\beta}{\chi} \sin (\chi t),
\end{aligned}
$$

where $\chi=\sqrt{q^{2} v_{s}^{2}+\beta^{2}}$ and $\hat{\phi}_{s}(t)=\eta \int_{0}^{t} \hat{I}_{p}\left(t^{\prime}\right) \mathrm{d} t^{\prime}$, with $\hat{I}_{p}(t)=\hat{\Psi}_{p}^{\dagger}(0, \tau) \hat{\Psi}_{p}(0, \tau)$. Thus all the spatial modes $\hat{\psi}_{ \pm}(q, t)=\int \mathrm{d} z e^{\mp i q z} \hat{\Psi}_{ \pm}(z, t)$ of the signal polariton acquire the same $q$ independent phase shift $\hat{\phi}_{s}(t)$. It follows from eqs. (2) that a signal polariton composed of modes with $|q| \ll \beta / v_{s}$ will be strongly trapped inside the medium, its wavepacket periodically cycling between the forward and backward components while interacting with the probe polariton, yielding

$$
\begin{aligned}
\hat{\Psi}_{+}(z, t) & =\hat{\Psi}_{+}(z, 0) e^{i \hat{\phi}_{s}(t)} \cos (\beta t), \\
\hat{\Psi}_{-}(z, t) & =i \hat{\Psi}_{+}(z, 0) e^{i \hat{\phi}_{s}(t)} \sin (\beta t), \\
\hat{\Psi}_{p}(z, t) & =\hat{\Psi}_{p}(0, \tau) e^{i \hat{\phi}_{p}(z)},
\end{aligned}
$$


where $\hat{\phi}_{p}(z)=\frac{\eta}{v_{p}} \int_{0}^{z} \hat{I}_{s}\left(z^{\prime}\right) \mathrm{d} z^{\prime}$, with $\hat{I}_{s}(z)=\Psi_{+}^{\dagger}(z, 0) \Psi_{+}(z, 0)$, is the probe phase-shift.

Let us dwell upon the approximations involved in the derivation of eqs. (3). During the conversion of the signal pulse into a standing-wave polaritonic excitation inside the periodic structure, the nonadiabatic corrections resulting in its dissipation are negligible provided the medium is optically thick, $\varsigma_{A} \rho_{A} L \gg 1$, where $\varsigma_{A}$ is the resonant absorption cross-section for the transition $|b\rangle_{A} \rightarrow\left|a^{\prime}\right\rangle_{A}$ and $\rho_{A}=N_{A} /(S L)$ is the density of atoms $A$ [6,7]. After the signal pulse has been trapped in the PBG, due to nonzero values of $q$, its gets spatially distorted (spreads) at a rate $\kappa_{d} \simeq q^{2} v_{s}^{2} /(\pi \beta)\left(0 \leq|q|<\beta / v_{s}\right)$. We can estimate the bandwidth of the signal pulse from its spatial extent as $\delta q \sim v_{s} /(c L)<\left|\Omega_{d}^{(A)}\right|^{2} /\left(\gamma_{a}^{(A)} c\right)$, thus obtaining the upper limit for the distortion rate $\kappa_{d} \leq 2 v_{s}^{3} /\left(\pi c L^{2} \delta \omega_{\mathrm{PBG}}\right)$. On the other hand, the bandwidth of the probe is limited by the length of the medium via $\delta \omega_{p}<\left|\Omega_{d}^{(B)}\right|^{2}\left[g_{B}^{2} N_{B} \gamma_{a}^{(B)} L / c\right]^{-1 / 2}=$ $\left|\Omega_{d}^{(B)}\right|{ }^{2} k_{p}\left[\gamma_{a}^{(B)} \sqrt{3 \pi / 2 \rho_{B} L}\right]^{-1}$, where $\rho_{B}=N_{B} /(S L)$ is the density of atoms $B$ [14]. Finally, the interaction time $t_{\text {int }}=L / v_{p}$ is limited by $t_{\text {int }} \times \max \left\{\kappa_{d}, \kappa_{s}, \kappa_{p}\right\} \ll 1$, and so the fidelity of the cross-phase modulation is given by $F=\exp \left[-\left(\kappa_{d}+\kappa_{s}+\kappa_{p}\right) L / v_{p}\right]$.

Consider first the classical limit of eqs. (3), where the operators $\hat{\Psi}_{p, \pm}$ and $\hat{I}_{p, s}$ are replaced by c-numbers. Then for two single-photon pulses $\frac{1}{L} \int I_{s} \mathrm{~d} z=\frac{v_{p}}{L} \int I_{p} \mathrm{~d} t \simeq 1$, the conditional phase shift accumulated by the probe and signal fields during the interaction is given by

$$
\phi_{p}=\phi_{s}=\frac{g_{B}^{\prime 2} L \cos ^{2} \theta_{A} \tan ^{2} \theta_{B}}{c \Delta_{B}} \equiv \phi .
$$

Note that the phase shift is proportional to the intensity of the photonic component of the signal polariton, as attested by the presence of the $\cos ^{2} \theta_{A}$ term in the nominator of eq. (4). For realistic experimental parameters, relevant to a doped periodic structure discussed below, one can obtain $\phi \simeq \pi$ (see fig. 1(c)) with the fidelity $F \simeq 1$.

We now turn to the fully quantum treatment of the system. To compare the classical and quantum pictures, we consider first the evolution of input wavepackets composed of the multimode coherent states $\left|\alpha_{p}\right\rangle \otimes\left|\alpha_{+}\right\rangle \otimes\left|0_{-}\right\rangle=\prod_{q_{p}}\left|\alpha_{p}^{q_{p}}\right\rangle \otimes \prod_{q}\left|\alpha_{+}^{q}\right\rangle \otimes \prod_{q^{\prime}}\left|0_{-}^{q^{\prime}}\right\rangle$. States $\left|\alpha_{p}\right\rangle$ and $\left|\alpha_{+}\right\rangle$are the eigenstates of the input fields operators $\hat{\mathcal{E}}_{p}(0, t)$ and $\hat{\mathcal{E}}_{+}(z, 0)$ with eigenvalues $\alpha_{p}(t)=\sum_{q_{p}} \alpha_{p}^{q_{p}} e^{-i q_{p} c t}$ and $\alpha_{+}(z)=\sum_{q} \alpha_{+}^{q} e^{i q z}$, respectively. The expectation values for the fields are then obtained as

$$
\begin{aligned}
\left\langle\hat{\mathcal{E}}_{p}(z, t)\right\rangle & =\alpha_{p}(\tau) \exp \left[\frac{e^{i \phi c / v_{s}}-1}{L} \int_{0}^{z}\left|\alpha_{+}\left(z^{\prime}\right)\right|^{2} \mathrm{~d} z^{\prime}\right] \\
\left\langle\hat{\mathcal{E}}_{ \pm}(z, t)\right\rangle & =\alpha_{+}(z) \exp \left[\frac{e^{i \phi}-1}{L} c \int_{0}^{t}\left|\alpha_{p}\left(\tau^{\prime}\right)\right|^{2} \mathrm{~d} t^{\prime}\right] \times\left[\begin{array}{c}
\cos (\beta t) \\
i \sin (\beta t)
\end{array}\right] .
\end{aligned}
$$

These equations are notably different from those obtained for single-mode [15] and multimode copropagating fields $[10,11]$ because all parts of the probe pulse interact with the whole signal pulse (and vice versa), as is manifest in the space (time) integration. Similarly to the cases discussed in $[10,11,15]$, eqs. (5]) reproduce the classical result only in the limit $\phi c / v_{s} \ll 1$, yielding $\phi_{p}=\frac{\eta c}{v_{p} v_{s}} \int\left|\alpha_{+}\right|^{2} \mathrm{~d} z^{\prime}$ and $\phi_{s}=\frac{\eta c}{v_{p}} \int\left|\alpha_{p}\right|^{2} \mathrm{~d} t^{\prime}$. A $\pi$ phase shift is then obtained for $\frac{c}{L v_{s}} \int\left|\alpha_{+}\right|^{2} \mathrm{~d} z^{\prime}=\frac{c}{L} \int\left|\alpha_{p}\right|^{2} \mathrm{~d} t^{\prime}=\pi / \phi$. This restriction on the classical correspondence of coherent states comes about since, for large enough cross-phase modulation rates $\eta$, these states exhibit periodic collapses and revivals as $\phi$ changes from 0 to $2 \pi$, which limits their usefulness for quantum information applications.

Consider finally the input state $\left|\Phi_{\text {in }}\right\rangle=\left|1_{p}\right\rangle \otimes\left|1_{+}\right\rangle \otimes\left|0_{-}\right\rangle$, consisting of two singleexcitation polariton wavepackets $\left|1_{p,+}\right\rangle=\sum_{q} \xi_{p,+}^{q}\left|1_{p,+}^{q}\right\rangle$, where the Fourier amplitudes, normalized as $\sum_{q}\left|\xi_{p,+}^{q}\right|^{2}=1$, define the spatial envelopes $f_{p,+}(z)=\sum_{q} \xi_{p,+}^{q} e^{i q z}$ of the probe 
and forward signal pulses that initially (at $t=0$ ) are localized around $z=0$ and $z=L / 2$, respectively (fig. 1(c)). During the evolution, the state of the system evolves according to $|\Phi(t)\rangle=\exp \left(-\frac{i}{\hbar} \int_{0}^{t} H_{\text {int }} \mathrm{d} t^{\prime}\right)\left|\Phi_{\text {in }}\right\rangle$, where $H_{\text {int }}=-\frac{\hbar}{L} \int \mathrm{d} z\left[\eta \hat{\Psi}_{p}^{\dagger} \hat{\Psi}_{p}\left(\hat{\Psi}_{+}^{\dagger} \hat{\Psi}_{+}+\hat{\Psi}_{-}^{\dagger} \hat{\Psi}_{-}\right)+\right.$ $\left.\beta\left(\hat{\Psi}_{+}^{\dagger} \hat{\Psi}_{-}+\hat{\Psi}_{-}^{\dagger} \hat{\Psi}_{+}\right)\right]$is the effective interaction Hamiltonian whose first and second terms commute. The implicit time-dependence of the effective Hamiltonian, due to the propagation of the probe-polariton pulse with the group velocity $v_{p}$, is contained in the operator $\hat{\Psi}_{p}=\hat{\Psi}_{p}(z-\zeta)$, with $\zeta=v_{p} t$. After the interaction, at time $t_{\text {out }}>L / v_{p}$, the output state of the system is

$$
\left|\Phi_{\text {out }}\right\rangle=e^{i \eta L / v_{p}}\left|1_{p}\right\rangle \otimes\left[\cos \left(\beta t_{\text {out }}\right)\left|1_{+}\right\rangle \otimes\left|0_{-}\right\rangle+i \sin \left(\beta t_{\text {out }}\right)\left|0_{+}\right\rangle \otimes\left|1_{-}\right\rangle\right],
$$

where $\left|1_{-}\right\rangle \equiv \sum_{q} \xi_{+}^{q}\left|1_{-}^{-q}\right\rangle$. Thus, while the signal pulse periodically cycles between the forward and backward modes, the combined state of the system acquires an overall conditional phase shift $\phi=\eta L / v_{p}$. When $\phi=\pi$, transformation (6) corresponds to the truth table of the universal controlled-phase (CPHASE) logic gate between the two photons representing qubits, which can be used to realize arbitrary unitary transformation [1].

Possible experimental realizations. - An $x-z$ 2D-periodic lattice of dielectric rods or semiconductor stacks (fig. 1(b)) [3,12,13], with controlled structural defects and mirror confinement in $y$, appears to be the most suitable structure for realizing polaritonic entanglement of two single-photons, since both the signal and the probe pulses may be confined in the $x-y$ directions in the vicinity of a "defect" row forming a PC waveguide, thereby avoiding diffraction losses and focusing the fields to a radius of $\sim 1 \mu \mathrm{m}[3,12]$. Using the double- $\Lambda$ dopants (atoms A), the signal pulse can be trapped in the PC, with the localization distance $z_{\text {loc }}$ extending over many periods, and interact with the probe pulse via atoms B. Expressing the atom-field coupling constants $g$ through the decay rate $\gamma$ of the corresponding excited state as $g=3 \pi c \gamma /\left(2 k^{2} S L\right)$, and assuming that $\gamma_{a}^{(A)} \simeq \gamma_{a}^{(B)}$ and $g_{A}^{2} N_{A} \gg\left|\Omega_{d}^{(A)}\right|^{2}\left(v_{s} \ll c\right)$, we have from eq. (41) $\phi \simeq 3 \pi \gamma_{d}\left(2 k_{p}^{2} \Delta_{B} S\right)^{-1}\left(\Omega_{d}^{(A)} / \Omega_{d}^{(B)}\right)^{2}\left(\rho_{B} / \rho_{A}\right)$. Among the possible dopants, III-V or II-VI $n$-doped semiconductor quantum dots, having large dipole moments and level structure conducive to EIT [16], could be the best choice for our scheme, provided high densities can be achieved. In such single-electron doped QDs, the spin degeneracy of the ground and the lowest and higher excited (charged exciton or trion) states can be lifted with a magnetic field [16], realizing the level scheme of fig. 1(a), where atoms A and B can spectroscopically be selected (via optical pumping or spectral hole burning) from the inhomogeneous ensemble of QDs. Thus states $|b\rangle$ and $|c\rangle$ are represented by the Zeeman-split spin-up and spin-down states of the conduction-band electron in the QD. The excited states $|a\rangle_{A}$ and $\left|a^{\prime}\right\rangle_{A}$ of atoms A can be the first and the second (or higher) exciton states, while states $|a\rangle_{B}$ and $|d\rangle_{B}$ of atoms B are the Zeeman sublevels of the lowest excitonic state. Assuming the parameters $L \simeq 0.1 \mathrm{~cm}, S \simeq 10^{-8} \mathrm{~cm}^{2}, \delta \omega_{\mathrm{PBG}} \sim 10^{14} \mathrm{rad} / \mathrm{s}[3,12], \rho_{A, B} \simeq 10^{12} \mathrm{~cm}^{-3}$, $\omega_{p} \simeq 3 \times 10^{15} \mathrm{rad} / \mathrm{s}, \Omega_{d}^{(A)} \simeq 5 \times 10^{8} \mathrm{rad} / \mathrm{s}, \Omega_{d}^{(B)} \simeq 2 \times 10^{7} \mathrm{rad} / \mathrm{s}, \Delta_{B} \simeq 10^{8} \mathrm{rad} / \mathrm{s}, \gamma_{a, d} \simeq 10^{7} \mathrm{~s}^{-1}$, and $\gamma_{b c} \simeq 10^{4} \mathrm{~s}^{-1}$ [16], we obtain $\phi \simeq \pi$ with the fidelity $F \geq 0.98 \%$, the main limiting factor being the decay of Raman coherence $\gamma_{b c}$. Other contenders for observing the proposed effects include periodic structures fabricated from rare-earth doped crystals, such as Pr:YSP, in which high-fidelity EIT has experimentally been demonstrated [17], or cryogenically cooled diamond with high density of nitrogen-vacancy defect centers [18].

Conclusions. - To summarize, we have proposed a new class of multimode quantum-field interactions involving quantized EIT-polaritons in PBG structures. We have shown that such interactions allow efficient cross-phase modulation between a propagating probe pulse and a 
trapped signal pulse, whose localization is achieved by an adiabatic four-wave mixing process that pulls its frequency into the PBG. This localization allows multiply repeated interaction of the signal with the entire probe pulse. As a result, the combined two-photon state of the system can acquire a conditional $\pi$ phase-shift, which corresponds to the universal CPHASE logic gate. The phase shift is spatially-uniform and the process may have high fidelity. The experimental realization of the predicted effects requires the fabrication of periodic structures with large densities of optically active dopants [16-18], which may also find useful applications in laser technology, optical communication or quantum computation. We note that a similar regime of giant cross-phase modulation with suppressed spectral broadening is also realizable in cold atomic vapors using optically-induced PBGs [19], on which we intend to report elsewhere. The proposed scheme may pave the way to quantum information applications such as deterministic all-optical quantum computation, dense coding and teleportation [1].

$$
* * *
$$

We gratefully acknowledge stimulating discussions with A. André and M. D. Lukin. This work was supported by the EC (QUACS, ATESIT and PHOREMOST Networks), ISF and Minerva.

\section{REFERENCES}

[1] Nielsen M. A. and Chuang I. L., Quantum Computation and Quantum Information (Cambridge University Press, Cambridge) 2000.

[2] Rauschenbeutel A. et al., Phys. Rev. Lett, 83 (1999) 5166; Knill E., Laflamme R. and Milburn G. J., Nature, 409 (2001) 46; Duan L.-M., Lukin M. D., CiraC J. I. and Zoller P., Nature, 414 (2001) 413.

[3] Johnson S. G. and Jonnnopoulos J. D., Photonic Crystals: The Road from Theory to Practice (Kluwer Academic, Boston) 2002; SAKodA K., Optical Properties of Photonic Crystals (Springer-Verlag, Berlin) 2001.

[4] Kurizki G. et al., Progress in Optics, edited by Wolf E., Vol. 42 (Elsevier, North-Holland) 2001, p. 93.

[5] Kurizki G. et al., J. Opt. Soc. Am. B, 19 (2002) 2066.

[6] Fleischhauer M. and Lukin M. D., Phys. Rev. Lett, 84 (2000) 5094; Fleischhauer M. and Lukin M. D., Phys. Rev. A, 65 (2002) 022314.

[7] Liu C., Dutton Z., Behroozi C. H. and Hau L.V., Nature, 409 (2001) 490; Phillips D. F. et al., Phys. Rev. Lett, 86 (2001) 783.

[8] Schmidt H. and ImamoĞLu A., Opt. Lett., 21 (1996) 1936.

[9] Harris S. and Hau L., Phys. Rev. Lett., 82 (1999) 4611.

[10] Lukin M. D. and ImamoĞLu A., Phys. Rev. Lett., 84 (2000) 1419.

[11] Petrosyan D. and Malakyan Yu. P., Phys. Rev. A, 70 (2004) 023822.

[12] Mingaleev S. F and Kivshar Yu. S., Phys. Rev. Lett., 86 (2001) 5474.

[13] Ogawa S. et al., Science, 305 (2004) 227.

[14] Lukin M. D. et al., Phys. Rev. Lett., 79 (1997) 2959.

[15] Sanders B. S and Milburn G. J., Phys. Rev. A, 45 (1992) 1919.

[16] Gammon D. and Steel D. G., Physics Today, 55(10) (2002) 36; ImamoĞlu A. et al., Phys. Rev. Lett., 83 (1999) 4204; KakKaWa J. M. et al., Science, 277 (1997) 1284; KaKkaWA J. M. and Awschalom D. D., Phys. Rev. Lett., 80 (1998) 4313.

[17] Turukhin A. V. et al., Phys. Rev. Lett., 88 (2001) 023602; Ham B., Hemmer P. and Shahriar M., Opt. Commun., 144 (1997) 227.

[18] Lenef A. et al., Phys. Rev. B, 53 (1996) 13427; 53 (1996) 13441.

[19] Andre A. and Lukin M. D., Phys. Rev. Lett., 89 (2002) 143602; Bajcsy M., Zibrov A. S. and Lukin M. D., Nature, 426 (2003) 638. 